\title{
gs \\ Landau level spectra and the quantum Hall effect of multilayer graphene
}

\author{
Mikito Koshino ${ }^{1}$ and Edward McCann ${ }^{2}$ \\ ${ }^{1}$ Department of Physics, Tohoku University, Sendai 980-8578, Japan \\ ${ }^{2}$ Department of Physics, Lancaster University, Lancaster LA1 4YB, United Kingdom
}

(Received 5 January 2011; published 27 April 2011)

\begin{abstract}
The Landau level spectra and the quantum Hall effect of ABA-stacked multilayer graphenes are studied in the effective-mass approximation. The low-energy effective-mass Hamiltonian may be partially diagonalized into an approximate block-diagonal form, with each diagonal block contributing parabolic bands except for an additional block describing Dirac-like bands with a linear dispersion in a multilayer with an odd number of layers. We fully include the band parameters and, taking into account the symmetry of the lattice, we analyze their effect on the block-diagonal Hamiltonian. Next-nearest-layer couplings are shown to be particularly important in determining the low-energy spectrum and the phase diagram of the quantum Hall conductivity by causing energy shifts, level anticrossings, and valley splitting of the low-lying Landau levels.
\end{abstract}

DOI: 10.1103/PhysRevB.83.165443

PACS number(s): 73.22.Pr, 81.05.ue, 73.43.Cd

\section{INTRODUCTION}

Since the isolation of graphene flakes, ${ }^{1}$ the chiral nature of quasiparticles in monolayer and bilayer graphene has been observed in a range of phenomena including the integer quantum Hall effect, ${ }^{2-4}$ Klein tunneling, ${ }^{5-8}$ weak localization, ${ }^{9-16}$ and photoemission. ${ }^{17-20}$ Generally speaking, the effective mass models ${ }^{21-30}$ of monolayer and bilayer graphene have been very successful in describing these phenomena. The low-energy band structure of bilayer graphene is composed of a pair of parabolic bands ${ }^{28-30}$ and is distinct from monolayer graphene, which has a Dirac-like linear dispersion. ${ }^{22,23}$ In a magnetic field, the Landau level structures of monolayer ${ }^{23-27}$ and bilayer ${ }^{28,31}$ graphene differ in the degeneracy at zero energy, and quantum Hall plateaus appear at different filling factors accordingly. ${ }^{2-4}$

In graphene multilayers with three or more layers, $N \geqslant 3$, where $N$ is the number of layers; the effective-mass model is much more complicated than in monolayer or bilayer graphene ${ }^{28,29}$ or even in bulk graphite. ${ }^{32}$ On the one hand, there are more relevant parameters than in monolayer or bilayer graphene because of the presence of next-nearest layer couplings; on the other hand, a lack of translational invariance in the direction of layer stacking means that the number of basis states in the model is $2 N$, not four as in the Slonczewski-WeissMcClure model of bulk graphite. ${ }^{32}$ Although multilayers with a moderate number of layers, $N \gtrsim 10$, are thought to be similar to bulk graphite ${ }^{33}$ the properties of few-layer graphene (typically $N \gtrsim 3$ ) are distinct. ${ }^{29,33-50}$

Here, we consider ABA-stacked (Bernal) multilayer graphene and analyze its Landau level spectrum and quantum Hall conductivity in magnetic fields. In the lattice structure in Figs. 1(a) and 1(b), the layers, each having two inequivalent atomic sites, $A$ and $B$, on a honeycomb lattice, are stacked so that half of the atomic sites have a counterpart directly above or below in the adjacent layers, referred to as dimer sites, whereas half the sites do not have such a partner (nondimer sites). The effective-mass model is characterized by the intralayer coupling $\gamma_{0}$, nearest interlayer couplings $\gamma_{1}, \gamma_{3}$, and $\gamma_{4}$, and next-nearest layer couplings $\gamma_{2}$ and $\gamma_{5}{ }^{21,22,28,29,32}$ By comparison with experiments, it has been possible to determine the values of relevant parameters, including intralayer coupling ${ }^{2,3,51,52}$ and, in bilayers, interlayer couplings. ${ }^{17,53-58}$

Although it is possible to numerically diagonalize an effective Hamiltonian with $2 N$ components, ${ }^{33-35}$ this approach does not always shed light on the roles of the different parameters. Nevertheless, it was noticed that the multilayer bands form groups of bilayerlike parabolic bands near the corners of the Brillouin zone called valleys ${ }^{59}$ with, in odd$N$ layers, an additional pair of monolayerlike bands with linear dispersion. ${ }^{29,33}$ Subsequently, it was realized that a partial diagonalization of the effective Hamiltonian could be performed in order to write it in block-diagonal form, ${ }^{36-38}$ as illustrated in Fig. 1(c). Each block on the diagonal describes four bilayerlike bands with a given effective mass (labeled $\mathrm{b}$ or $\mathrm{B})$ or, in odd- $N$ layers, two monolayerlike bands (labeled M).

The decomposition into block-diagonal form is based upon the eigenstates of a one-dimensional tight-binding chain in the stacking direction (perpendicular to the layers) with nearestneighbor hopping. ${ }^{29}$ If the next-nearest layer couplings, $\gamma_{2}$ and $\gamma_{5}$, are neglected, the decomposition is exact: the diagonal blocks (and their corresponding bands) are completely separate from each other. The matrix elements associated with the next-nearest layer couplings appear within each block of the decomposed Hamiltonian, but they also couple separate blocks [as shown by the $\mathcal{W}$ blocks in Fig. 1(c)] and tend to hybridize their bands. ${ }^{38}$ In this paper we calculate the Landau levels with the band parameters fully included and show with the aid of decomposition that the next-nearest-neighbor couplings generally account for the energy shifts, the level anticrossings, and valley splitting of the low-lying Landau levels, which significantly influence the phase diagram of the quantum Hall conductivity. We focus on trilayer, four-layer, and five-layer graphene (Fig. 1) as representative examples.

\section{THE EFFECTIVE-MASS MODEL OF MULTILAYER GRAPHENE}

\section{A. The effective-mass Hamiltonian}

To describe the electronic properties of Bernal-stacked multilayer graphene, we use an effective-mass model with the 
(a)
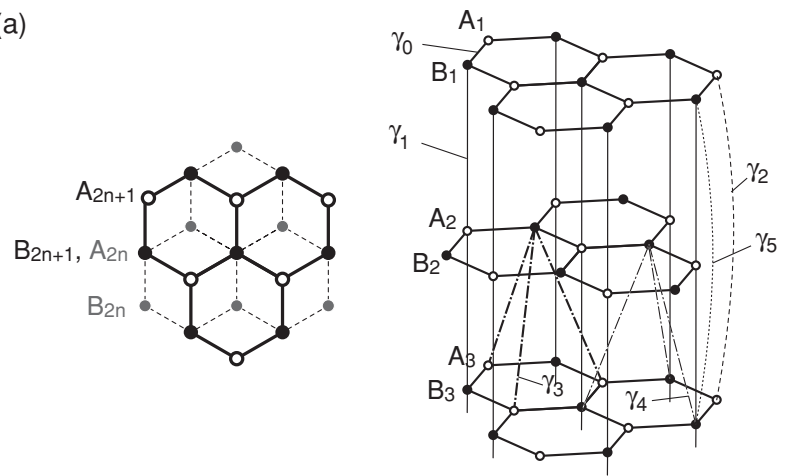

(b) Trilayer

Fourlayer

Fivelayer
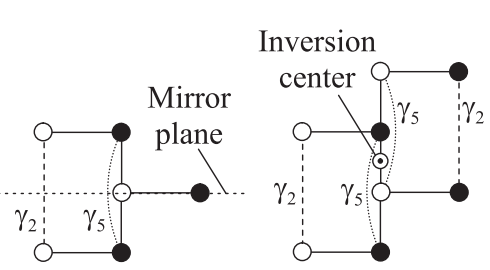

(c)

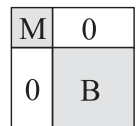

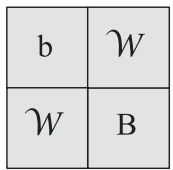
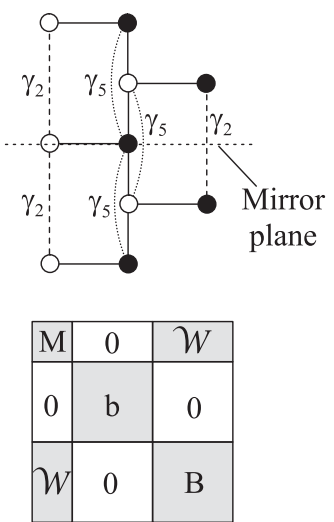

FIG. 1. (a) Atomic structure of ABA-stacked multilayer graphene. (b) Side view of (left) trilayer, (middle) four-layer, and (right) five-layer lattices, showing the mirror planes for odd- $N$ layers and the inversion center for even- $N$ layers. Horizontal solid lines indicate $\gamma_{0}$ intralayer coupling between $A$ (white) and $B$ (black) atoms; vertical solid lines represent $\gamma_{1}$. (c) Schematic form of the partially diagonalized Hamiltonians with $\mathrm{M}, \mathrm{b}$, and $\mathrm{B}$ indicating monolayerlike, light bilayerlike, and heavy bilayerlike blocks, respectively; $\mathcal{W}$ represents coupling between them arising from next-nearest layer couplings, $\gamma_{2}$ and $\gamma_{5}$.

Slonczewski-Weiss-McClure parametrization of graphite. ${ }^{32}$ The low energy spectrum is given by states in the vicinity of the $K_{\xi}$ point in the Brillouin zone, where $\xi= \pm 1$ is the valley index. If $\left|A_{j}\right\rangle$ and $\left|B_{j}\right\rangle$ are Bloch functions at the $K_{\xi}$ point, corresponding to the $A$ and $B$ sublattices of layer $j$, respectively, then a suitable basis is $\left|A_{1}\right\rangle,\left|B_{1}\right\rangle ;\left|A_{2}\right\rangle,\left|B_{2}\right\rangle$; $\ldots ;\left|A_{N}\right\rangle,\left|B_{N}\right\rangle$. In this basis, the Hamiltonian of multilayer graphene with $N$ layers $^{29,33,34,36,38}$ in the vicinity of the $K_{\xi}$ valley is

$$
H_{N}=\left(\begin{array}{ccccccc}
H_{0} & V & W & & & & \\
V^{\dagger} & H_{0}^{\prime} & V^{\dagger} & W^{\prime} & & & \\
W & V & H_{0} & V & W & & \\
& W^{\prime} & V^{\dagger} & H_{0}^{\prime} & V^{\dagger} & W^{\prime} & \\
& & \ddots & \ddots & \ddots & \ddots & \ddots
\end{array}\right)
$$

with

$$
H_{0}=\left(\begin{array}{cc}
0 & v \pi^{\dagger} \\
v \pi & \Delta^{\prime}
\end{array}\right), \quad H_{0}^{\prime}=\left(\begin{array}{cc}
\Delta^{\prime} & v \pi^{\dagger} \\
v \pi & 0
\end{array}\right)
$$

$$
\begin{gathered}
V=\left(\begin{array}{cc}
-v_{4} \pi^{\dagger} & v_{3} \pi \\
\gamma_{1} & -v_{4} \pi^{\dagger}
\end{array}\right), \\
W=\left(\begin{array}{ll}
\gamma_{2} / 2 & 0 \\
0 & \gamma_{5} / 2
\end{array}\right), \quad W^{\prime}=\left(\begin{array}{cc}
\gamma_{5} / 2 & 0 \\
0 & \gamma_{2} / 2
\end{array}\right) .
\end{gathered}
$$

Here, the in-plane momentum operator is $\pi=\xi p_{x}+i p_{y}$, and $\boldsymbol{p}=\left(p_{x}, p_{y}\right)=-i \hbar \nabla+e \mathbf{A}$ with vector potential $\mathbf{A}$. The diagonal blocks, Eq. (2), describe nearest-neighbor intralayer coupling, and $V$, Eq. (3), describes nearest-neighbor layer coupling, where $\gamma_{1}$ is the interlayer coupling between dimer sites. Parameter $\Delta^{\prime}$ represents the energy difference between dimer sites and nondimer sites, and thus it only exists for $N \geqslant$ 2. It is related to the band parameters as $\Delta^{\prime}=\Delta-\gamma_{2}+\gamma_{5}$. The Fermi velocity of monolayer graphene is $v=\sqrt{3} a \gamma_{0} / 2 \hbar$, and other velocities are defined as $v_{3}=\sqrt{3} a \gamma_{3} / 2 \hbar$ and $v_{4}=$ $\sqrt{3} a \gamma_{4} / 2 \hbar$. Matrix $W$, Eq. (4), describes coupling between next-nearest-neighboring layers, and it only exists for $N \geqslant 3$. Parameters $\gamma_{2}$ and $\gamma_{5}$ couple a pair of nondimer sites and a pair of dimer sites, respectively.

\section{B. Decomposition to an approximate block-diagonal form}

The decomposition of the Hamiltonian, Eq. (1), into an approximate block-diagonal form uses a unitary transformation based on the eigenstates of a linear chain of atoms in the $z$ direction: ${ }^{36-38}$

$$
\begin{aligned}
& f_{m}(j)=c_{m} \sqrt{\frac{2}{N+1}}\left[1-(-1)^{j}\right] \sin \kappa_{m} j, \\
& g_{m}(j)=c_{m} \sqrt{\frac{2}{N+1}}\left[1+(-1)^{j}\right] \sin \kappa_{m} j,
\end{aligned}
$$

where

$$
\begin{gathered}
\kappa_{m}=\frac{\pi}{2}-\frac{m \pi}{2(N+1)}, \\
c_{m}= \begin{cases}1 / 2 & (m=0), \\
1 / \sqrt{2} & (m \neq 0) .\end{cases}
\end{gathered}
$$

Here $j=1,2, \ldots, N$ is the layer index, and $m$ is the block index, which ranges as

$$
m=\left\{\begin{array}{l}
1,3,5, \ldots, N-1, \quad N=\text { even }, \\
0,2,4, \ldots, N-1, \quad N=\text { odd } .
\end{array}\right.
$$

Obviously $f_{m}(j)$ is zero on even $j$ layers, while $g_{m}(j)$ is zero on odd $j$ layers. The basis is constructed ${ }^{36,38}$ by assigning $f_{m}(j), g_{m}(j)$ to each site as

$$
\begin{aligned}
\left|\phi_{m}^{(X, \text { odd })}\right\rangle & =\sum_{j=1}^{N} f_{m}(j)\left|X_{j}\right\rangle, \\
\left|\phi_{m}^{(X, \text { even })}\right\rangle & =\sum_{j=1}^{N} g_{m}(j)\left|X_{j}\right\rangle,
\end{aligned}
$$

where $X=A$ or $B$. A superscript such as (A, odd) indicates that the wave function has a nonzero amplitude only on $\left|A_{j}\right\rangle$ sites with odd $j$ 's.

In order to write the Hamiltonian Eq. (1) in terms of the basis states Eq. (10), we group the basis of block $m$ 
as $\mathbf{u}_{m}=\left\{\left|\phi_{m}^{(A, \text { odd })}\right\rangle,\left|\phi_{m}^{(B, \text { odd })}\right\rangle,\left|\phi_{m}^{(A, \text { even })}\right\rangle,\left|\phi_{m}^{(B, \text { even })}\right\rangle\right\}$. Then, the block matrix between different $m$ 's may be written as

$$
\mathcal{H}_{m^{\prime} m} \equiv \mathbf{u}_{m^{\prime}}^{\dagger} H \mathbf{u}_{m}=\mathcal{H}\left(\lambda_{m}\right) \delta_{m^{\prime} m}+\mathcal{W}\left(\alpha_{m^{\prime} m}, \beta_{m^{\prime} m}\right)
$$

with

$$
\begin{gathered}
\mathcal{H}(\lambda)=\left(\begin{array}{lccc}
0 & v \pi^{\dagger} & -\lambda v_{4} \pi^{\dagger} & \lambda v_{3} \pi \\
v \pi & \Delta^{\prime} & \lambda \gamma_{1} & -\lambda v_{4} \pi^{\dagger} \\
-\lambda v_{4} \pi & \lambda \gamma_{1} & \Delta^{\prime} & v \pi^{\dagger} \\
\lambda v_{3} \pi^{\dagger} & -\lambda v_{4} \pi & v \pi & 0
\end{array}\right) \\
\mathcal{W}(\alpha, \beta)=\left(\begin{array}{cccc}
\alpha \gamma_{2} & 0 & 0 & 0 \\
0 & \alpha \gamma_{5} & 0 & 0 \\
0 & 0 & \beta \gamma_{5} & 0 \\
0 & 0 & 0 & \beta \gamma_{2}
\end{array}\right)
\end{gathered}
$$

where

$$
\begin{gathered}
\lambda_{m}=2 \cos \kappa_{m}, \\
\alpha_{m^{\prime} m}=2 c_{m} c_{m^{\prime}}\left(\delta_{m m^{\prime}}\left(1+\delta_{m 0}\right) \cos 2 \kappa_{m}\right. \\
\left.+\frac{\sin \kappa_{m} \sin \kappa_{m^{\prime}}}{N+1}\left\{2+(-1)^{\frac{m-m^{\prime}}{2}}\left[1-(-1)^{N}\right]\right\}\right), \\
\beta_{m^{\prime} m}=2 c_{m} c_{m^{\prime}}\left\{\delta_{m m^{\prime}}\left(1-\delta_{m 0}\right) \cos 2 \kappa_{m}\right. \\
\left.+\frac{\sin \kappa_{m} \sin \kappa_{m^{\prime}}}{N+1}(-1)^{\frac{m-m^{\prime}}{2}}\left[1+(-1)^{N}\right]\right\} .
\end{gathered}
$$

The diagonal matrix $\mathcal{H}_{m m}$ is equivalent to the Hamiltonian of bilayer graphene ${ }^{28}$ with nearest-layer coupling parameters multiplied by $\lambda,{ }^{29,33,36-38}$ and onsite asymmetric potential described by $\mathcal{W}_{m m}$. The off-diagonal block, $\mathcal{W}$ for $m \neq m^{\prime}$, has not been explicitly obtained before: it appears only when coupling between the next-nearest-neighboring layers, $\gamma_{2}$ and $\gamma_{5}$, is nonzero. The block $\mathcal{W}$ is diagonal, where $\gamma_{2}$ only connects pairs of nondimer sites, and $\gamma_{5}$ only connects pairs of dimer sites, of these effective bilayerlike blocks.

The case of $m=0$ is special in that $g_{m}(j)$ is identically zero, so only two basis states $\left\{\left|\phi_{0}^{(A, \text { odd })}\right\rangle,\left|\phi_{0}^{(B, \text { odd })}\right\rangle\right\}$ survive in Eq. (10). The matrix elements associated with the two missing basis states should be neglected in Eqs. (12) and (13). Specifically, the matrix for the $m=0$ block written in the two-component basis is ${ }^{38}$

$$
\mathcal{H}_{0}=\left(\begin{array}{cc}
0 & v \pi^{\dagger} \\
v \pi & \Delta^{\prime}
\end{array}\right)-\frac{N-1}{N+1}\left(\begin{array}{cc}
\gamma_{2} & 0 \\
0 & \gamma_{5}
\end{array}\right)
$$

which, barring the diagonal terms, is equivalent to the Hamiltonian of monolayer graphene.

We stress the role of the symmetry of the lattice and note that the even-odd effect, with respect to the number of layers, goes further than the absence or presence of monolayerike bands in the band structure. The lattice of odd- $N$ multilayers obeys mirror reflection symmetry $(x, y, z) \rightarrow(x, y,-z)$ (see Refs. 35, 36, 42, 45, and 60) [mirror planes for trilayer and five-layer graphene are shown in Fig. 1(b)], and thus the eigenstates can be classified by parity with respect to the reflection: the parity of the wave function of the subband $m$ is given by $(-1)^{\frac{N-m-1}{2}}$, so the group of $m=2,6,10, \ldots$ and that of $m=0,4,8, \ldots$ have opposite parities. Since eigenstates with different parity cannot be mixed by terms in the Hamiltonian that preserve lattice symmetry, off-diagonal blocks connecting diagonal blocks with different parity are identically zero even in the presence of next-nearest layer couplings. ${ }^{38,42} \mathrm{We}$ actually see that the coupling matrix $\mathcal{W}_{m m^{\prime}}$ between blocks having different parities indeed vanishes, as illustrated in Fig. 1(c) for trilayer and five-layer graphene.

Even- $N$ multilayers lack mirror reflection symmetry, however, so $\gamma_{2}$ and $\gamma_{5}$ mix every diagonal block, as shown in Fig. 1(c) for four-layer graphene. Instead, the lattice of even- $N$ multilayers obeys spatial inversion symmetry $(x, y, z) \rightarrow(-x,-y,-z)$ (see Refs. 35, 36, 42, 45, and 60) (an inversion center for four-layer graphene is shown in Fig. 1, center). Unlike mirror reflection, inversion symmetry transforms electronic states between valleys, and, even in the presence of a magnetic field, this ensures degeneracy of the electronic spectra at different valleys. ${ }^{45}$

\section{Reduced low-energy Hamiltonian}

As we show below, mixing between blocks is particularly important in the vicinity of level crossings. Level crossings aside, a good approximation to the spectra over a broad range of energy may be obtained by neglecting the off-diagonal blocks. ${ }^{38}$ Taken alone, the bilayerlike block $\mathcal{H}_{m m}$ for $m \neq 0$ describes four bands, ${ }^{28}$ two split off by energy $\pm \lambda \gamma_{1}$ at the $K_{\xi}$ point and two near zero energy. The split bands can be viewed as a bonding and antibonding pair created by the relatively strong interlayer coupling $\gamma_{1}$ between dimer sites (A, even) and (B, odd). For low energy, $\epsilon \ll \lambda \gamma_{1}$, it is possible to derive a reduced Hamiltonian for the bilayerlike block describing an effective hopping between nondimer sites (A, odd) and (B, even) by using a Schrieffer-Wolff transformation ${ }^{28,61}$ to eliminate components $\left|\phi_{m}^{(A \text {,even })}\right\rangle$ and $\left|\phi_{m}^{(B, \text { odd })}\right\rangle$. Then the basis of block $m \neq 0$ is reduced to $\widetilde{\mathbf{u}}_{m}=\left\{\left|\phi_{m}^{(A, \text { odd })}\right\rangle,\left|\phi_{m}^{(B, \text { even })}\right\rangle\right\}$ and the Hamiltonian matrix for the block is modified as

$$
\begin{gathered}
\tilde{\mathcal{H}}_{m m}=\tilde{\mathcal{H}}\left(\lambda_{m}\right)+\tilde{\mathcal{W}}\left(\alpha_{m m}, \beta_{m m}\right), \\
\tilde{\mathcal{H}}(\lambda)=-\frac{v^{2}}{\lambda \gamma_{1}}\left(\begin{array}{cc}
0 & \left(\pi^{\dagger}\right)^{2} \\
\pi^{2} & 0
\end{array}\right)+\lambda v_{3}\left(\begin{array}{ll}
0 & \pi \\
\pi^{\dagger} & 0
\end{array}\right) \\
+\frac{2 v v_{4}}{\gamma_{1}}\left(\begin{array}{cc}
\pi^{\dagger} \pi & 0 \\
0 & \pi \pi^{\dagger}
\end{array}\right), \\
\tilde{\mathcal{W}}(\alpha, \beta)=\left(\begin{array}{cc}
\alpha \gamma_{2} & 0 \\
0 & \beta \gamma_{2}
\end{array}\right) .
\end{gathered}
$$

This reduced Hamiltonian is approximately valid at low energy, $\{\epsilon, v p\} \ll \lambda \gamma_{1}$.

When $v_{3}$ and $v_{4}$ are neglected, the eigenvalues of $\tilde{\mathcal{H}}_{m m}$ at zero magnetic field are

$$
\epsilon_{ \pm}^{(m)}(p)=\frac{\alpha+\beta}{2} \gamma_{2} \pm \sqrt{\left(\frac{\alpha-\beta}{2} \gamma_{2}\right)^{2}+\frac{v^{4} p^{4}}{\left(\lambda \gamma_{1}\right)^{2}}},
$$


with $\lambda=\lambda_{m}, \alpha=\alpha_{m m}$, and $\beta=\beta_{m m}$. This gives nearly parabolic conduction and valence bands centered at energy $(\alpha+\beta) \gamma_{2} / 2$ with an energy gap $\left|(\alpha-\beta) \gamma_{2}\right|$ between them. The gap always vanishes for even-layered graphene since $\alpha_{m m}=\beta_{m m}$ holds for all $m$ when $N$ is even. The extra parameter $v_{3}$ introduces trigonal warping in a similar manner as in bilayer graphene, ${ }^{28}$ and the $v_{4}$ parameter produces a weak electron-hole asymmetry by adding the band energy $2 v v_{4} p^{2} / \gamma_{1}$ in both conduction and valence bands. ${ }^{62}$

The Landau level spectrum in a uniform and perpendicular magnetic field may be found using the Landau gauge $\mathbf{A}=(0, B x, 0)$. Then, at valley $K_{+}$, the operators $\pi$ and $\pi^{\dagger}$ coincide with raising and lowering operators ${ }^{63}$ in the basis of Landau functions $\psi_{n}(x, y)=e^{i p_{y} y / \hbar} \phi_{n}(x-$ $\left.p_{y} \lambda_{B}^{2}\right)$, such that $\pi \psi_{n}=i\left(\hbar / \lambda_{B}\right) \sqrt{2(n+1)} \psi_{n+1}, \pi^{\dagger} \psi_{n}=$ $-i\left(\hbar / \lambda_{B}\right) \sqrt{2 n} \psi_{n-1}$, and $\pi^{\dagger} \psi_{0}=0$. Here $\lambda_{B}=\sqrt{\hbar /(e B)}$ is the magnetic length. At valley $K_{-}$, the effect of the operators becomes $\pi^{\dagger} \psi_{n}=-i\left(\hbar / \lambda_{B}\right) \sqrt{2(n+1)} \psi_{n+1}, \pi \psi_{n}=$ $i\left(\hbar / \lambda_{B}\right) \sqrt{2 n} \psi_{n-1}$, and $\pi \psi_{0}=0$. In the absence of $v_{3}$ and $v_{4}$, the Landau level spectrum for the Hamiltonian $\widetilde{\mathcal{H}}_{m m}$ at the valley $\xi$ is given by

$$
\begin{gathered}
\epsilon_{n \geqslant 1, \pm}^{(m)}=\frac{\alpha+\beta}{2} \gamma_{2} \pm \sqrt{\left(\frac{\alpha-\beta}{2} \gamma_{2}\right)^{2}+\frac{n(n+1) \Gamma_{B}^{4}}{\left(\lambda \gamma_{1}\right)^{2}},} \\
\epsilon_{n=-1}^{(m)}=\epsilon_{n=0}^{(m)}=\left(\frac{1+\xi}{2} \alpha+\frac{1-\xi}{2} \beta\right) \gamma_{2},
\end{gathered}
$$

where $\Gamma_{B}=\sqrt{2 \hbar v^{2} e B}=\sqrt{2} \hbar v / \lambda_{B}$ and we consider $\left\{|\epsilon|, \sqrt{n} \Gamma_{B}\right\} \ll\left|\gamma_{1}\right|$. When $\alpha \neq \beta$, each of two lowest levels at $n=-1,0$ splits in valleys due to next-layer coupling $\gamma_{2}$, moving to energy corresponding to either the bottom of the zero-field conduction band or the top of the valence band. Other levels are valley degenerate in this approximation.

Even the degeneracy of the $n=0$ and $n=-1$ levels is lifted in the higher order of $\Gamma_{B} /\left(\lambda \gamma_{1}\right)$. By applying a perturbation to the original $4 \times 4$ Hamiltonian, we find the correction to be

$$
\begin{aligned}
\delta \epsilon_{n=-1}^{(m)}= & 0, \\
\delta \epsilon_{n=0}^{(m)}= & {\left[\left(\frac{1+\xi}{2} \alpha+\frac{1-\xi}{2} \beta\right) \gamma_{5}+\Delta^{\prime}+\frac{2 \lambda v_{4}}{v} \lambda \gamma_{1}\right] } \\
& \times \frac{\Gamma_{B}^{2}}{\left(\lambda \gamma_{1}\right)^{2}},
\end{aligned}
$$

so the splitting is proportional to $B$.

The monolayerlike block for $m=0$, Eq. (17), is characterized by only one parameter with $\alpha=-(N-1) /(N+1)$. The energy dispersion is given by

$$
\begin{aligned}
\epsilon_{ \pm}^{(0)}(p)= & \frac{\Delta^{\prime}+\alpha\left(\gamma_{2}+\gamma_{5}\right)}{2} \\
& \pm \sqrt{\left(\frac{\Delta^{\prime}-\alpha\left(\gamma_{2}-\gamma_{5}\right)}{2}\right)^{2}+v^{2} p^{2}},
\end{aligned}
$$

which generally has an energy gap of the width $\mid \Delta^{\prime}-$ $\alpha\left(\gamma_{2}-\gamma_{5}\right) \mid$ at the Dirac point and an overall energy shift of $\left[\Delta^{\prime}+\alpha\left(\gamma_{2}+\gamma_{5}\right)\right] / 2$. In a magnetic field, Landau levels become $^{64}$

$$
\begin{aligned}
\epsilon_{n \geqslant 1, \pm}^{(0)}= & \frac{\Delta^{\prime}+\alpha\left(\gamma_{2}+\gamma_{5}\right)}{2} \\
& \pm \sqrt{\left(\frac{\Delta^{\prime}-\alpha\left(\gamma_{2}-\gamma_{5}\right)}{2}\right)^{2}+n \Gamma_{B}^{2},} \\
\epsilon_{n=0}^{(0)}= & \frac{1+\xi}{2} \alpha \gamma_{2}+\frac{1-\xi}{2}\left(\Delta^{\prime}+\alpha \gamma_{5}\right) .
\end{aligned}
$$

Similarly to the bilayerlike block, the lowest levels at $n=0$ of $K_{+}$and $K_{-}$split to the energy of the bottom of the zero-field conduction band or to the top of the valence band, while the other levels are valley degenerate.

The Landau levels of the different blocks are hybridized by the off-diagonal matrix $\mathcal{W}_{m m^{\prime}}$. At the valley $K_{+}$the wave function of the Landau level with index $n$ can be written in the form $\left(c_{1} \psi_{n-1}, c_{2} \psi_{n}, c_{3} \psi_{n}, c_{4} \psi_{n+1}\right)$ for the bilayerlike band, and $\left(c_{1} \psi_{n-1}, c_{2} \psi_{n}\right)$ for the monolayerlike band. Since $\mathcal{W}_{m m^{\prime}}$ is diagonal and does not include $\pi$ or $\pi^{\dagger}$, it only couples Landau levels of different blocks if they have the same index $n$. Furthermore, parameter $\gamma_{3}$ mixes levels $n$ and $n+3$ within the same block, and thus it, together with $\mathcal{W}_{m m^{\prime}}$, leads to hybridization among the levels of different blocks whose indices are equal in modulo 3 . Such coupling leads to anticrossing at the intersecting point of the corresponding Landau levels. At the valley $K_{-}$, the wave function of the Landau level with index $n$ becomes $\left(c_{1} \psi_{n+1}, c_{2} \psi_{n}, c_{3} \psi_{n}, c_{4} \psi_{n-1}\right)$ for the bilayerlike band and $\left(c_{1} \psi_{n}, c_{2} \psi_{n-1}\right)$ for the monolayerlike band, where the index at the same position differs between monolayer and bilayer. As a result, the above rule for $K_{+}$changes only for the coupling between monolayer and bilayer levels, where the $n$th monolayer level couples with the $n^{\prime}$ th bilayer level when $n-1$ and $n^{\prime}$ are equal in modulo 3 .

\section{TRILAYER GRAPHENE}

According to the decomposition described previously, the Hamiltonian in basis $\left|\phi_{0}^{(A, \text { odd })}\right\rangle,\left|\phi_{0}^{(B, \text { odd })}\right\rangle,\left|\phi_{2}^{(A, \text { odd })}\right\rangle,\left|\phi_{2}^{(B, \text { odd })}\right\rangle$, $\left|\phi_{2}^{(A, \text { even })}\right\rangle,\left|\phi_{2}^{(B, \text { even })}\right\rangle$ may be written in block-diagonal form ${ }^{42}$ as

$$
H_{N=3}=\left(\begin{array}{cc}
\mathcal{H}_{0} & 0 \\
0 & \mathcal{H}_{2}
\end{array}\right)
$$

where

$$
\begin{gathered}
\mathcal{H}_{0}=\left(\begin{array}{cc}
0 & v \pi^{\dagger} \\
v \pi & \Delta^{\prime}
\end{array}\right)-\frac{1}{2}\left(\begin{array}{cc}
\gamma_{2} & 0 \\
0 & \gamma_{5}
\end{array}{ }^{\prime}\right) \\
\mathcal{H}_{2}=\mathcal{H}\left(\lambda_{2}\right)+\mathcal{W}(1 / 2,0),
\end{gathered}
$$

where $\lambda_{2}=\sqrt{2}$. The off-diagonal blocks in Eq. (26) connecting the monolayerlike block $\mathcal{H}_{0}$ and the bilayerlike block $\mathcal{H}_{2}$ are identically zero, because the basis states for the monolayerlike and bilayerlike blocks have different parity with respect to mirror reflection, as argued above.

In the absence of $v_{3}$ and $v_{4}$, the low-energy Landau level spectrum for the bilayerlike band $(m=2)$ is given by Eq. (22) with $(\lambda, \alpha, \beta)=(\sqrt{2}, 1 / 2,0)$, and that for the 
monolayerlike band ( $m=0$ ) by Eq. (25) with $\alpha=-1 / 2$. The lowest Landau levels of each block $\epsilon_{n=0}^{(0)}, \epsilon_{n=-1}^{(2)}$, and $\epsilon_{n=0}^{(2)}$, which are degenerate in the absence of next-layer coupling, ${ }^{29}$ split according to

$$
\begin{gathered}
\epsilon_{n=0}^{(0)}=\frac{1+\xi}{2}\left(-\frac{\gamma_{2}}{2}\right)+\frac{1-\xi}{2}\left(\Delta^{\prime}-\frac{\gamma_{5}}{2}\right), \\
\epsilon_{n=-1}^{(2)}=\epsilon_{n=0}^{(2)}=(1+\xi) \frac{\gamma_{2}}{4} .
\end{gathered}
$$

The two lowest levels of the bilayerlike block, $\epsilon_{n=-1}^{(2)}, \epsilon_{n=0}^{(2)}$, are weakly split by extra band parameters in accordance with Eq. (23). As a result, the 12 zero-energy levels split into six different energies, each of them having twofold spin degeneracy.

We numerically calculate the Landau level spectrum by diagonalizing the original Hamiltonian Eq. (1) including all parameters. We adopt the parameter values ${ }^{32} \gamma_{2}=-0.02 \mathrm{eV}$, $\gamma_{5}=0.04 \mathrm{eV}, \Delta^{\prime}=0.05 \mathrm{eV}, \gamma_{0}=3 \mathrm{eV}, \gamma_{1}=0.4 \mathrm{eV}, \gamma_{3}=$ $0.3 \mathrm{eV}$, and $\gamma_{4}=0.04 \mathrm{eV}$. Since the dimension of the Hamiltonian matrix becomes infinite in the presence of trigonal warping, we introduce a cutoff in the Landau level index, $n=100$, which is high enough to obtain the proper low-energy spectrum. $^{62}$

Figures 2(a) and 2(b) show the zero-field dispersion and the Landau levels plotted against the magnetic field, respectively, of ABA-trilayer graphene with all the band parameters included. The symbols $\mathrm{M}$ and $\mathrm{B}$ represent the monolayer block $(m=0)$ and the bilayer block $(m=2)$, respectively. The spectrum is composed of monolayerlike and bilayerlike Landau levels that are shifted relative to each other in energy, as qualitatively described by the Hamiltonian decomposition above, Eqs. (26)-(28). Zero energy levels are close to those obtained analytically, Eqs. (29) and (30), corresponding to the bottom of the zero-field conduction band or to the top of the valence band. We also observe the weak splitting of $\epsilon_{n=-1}^{(2)}, \epsilon_{n=0}^{(2)}$ [indicated as $(\mathrm{B},-1),(\mathrm{B}, 0)$, respectively] argued above.

Figure 2(c) shows a two-dimensional plot of the density of states in the space of magnetic field and the carrier density. For simplicity, we assume that each Landau level is broadened into a Gaussian shape with width $\Delta E=C \Gamma_{B},{ }^{24,65}$ where the constant $C$ is taken to be 0.03 . The dark and light shading (dark and bright colors) represent low and high densities of states, respectively. Apart from the usual Landau fan, we observe a series of bright lines corresponding to the crossings of monolayerlike and bilayerlike Landau levels. ${ }^{66}$ The numbers assigned to the dark regions indicate the quantized Hall conductivity in units of $e^{2} / h$ and correspond to those assigned to spaces between Landau levels in Fig. 2(b). The quantized Hall conductivity jumps by 2 only at the zero levels $\epsilon_{n=0}^{(0)}$, $\epsilon_{n=-1}^{(2)}, \epsilon_{n=0}^{(2)}$, which split into valleys, while it changes in units of 4 otherwise since other levels are almost valley degenerate. As a result, the quantized Hall conductivity becomes $4 M$ (where $M$ is an integer) only in the region between $\epsilon_{n=0}^{(0)}$ levels of two valleys, corresponding to the energy gap of the monolayer band, and also in the narrow regions between $\epsilon_{n=-1}^{(2)}$ and $\epsilon_{n=0}^{(2)}$ for each of $K_{+}$and $K_{-}$. Otherwise, it takes a series of $4 M+2$.
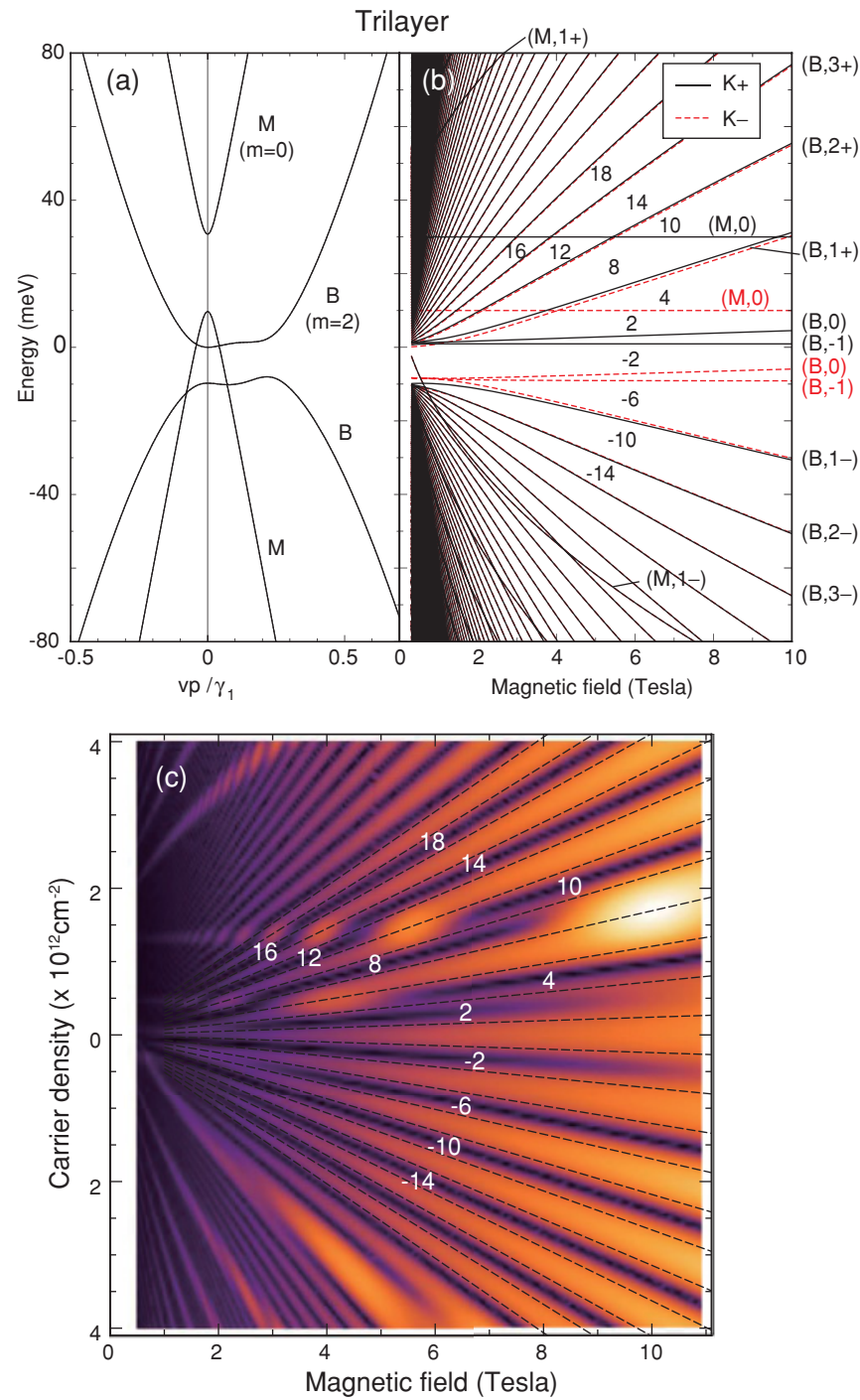

FIG. 2. (Color online) (a) Low-energy band structure and (b) Landau levels as a function of magnetic field of ABA-trilayer graphene. (c) Two-dimensional density plot of the density of states in the space of magnetic field and carrier density. In (b) and (c), numbers represent the quantized Hall conductivity in units of $e^{2} / h$.

\section{FOUR-LAYER GRAPHENE}

The Hamiltonian of ABA-stacked four-layer Hamiltonian, Eq. (1) for $N=4$, may be partially decomposed into two bilayerlike blocks with subsystem indices $m=1$ and $m=3$ :

$$
H_{N=4}=\left(\begin{array}{ll}
\mathcal{H}_{1} & \mathcal{H}_{13} \\
\mathcal{H}_{31} & \mathcal{H}_{3}
\end{array}\right)
$$

where

$$
\begin{gathered}
\mathcal{H}_{1}=\mathcal{H}\left(\lambda_{1}\right)+\mathcal{W}(-p,-p), \\
\mathcal{H}_{3}=\mathcal{H}\left(\lambda_{3}\right)+\mathcal{W}(p, p), \\
\mathcal{H}_{13}=\mathcal{H}_{31}=\mathcal{W}(p / 2,-p / 2) .
\end{gathered}
$$

and $\lambda_{1}=(-1+\sqrt{5}) / 2, \lambda_{3}=(1+\sqrt{5}) / 2$, and $p=1 / \sqrt{5}$.

By neglecting the interblock mixing as well as $v_{3}$ and $v_{4}$, the low-energy Landau level spectrum is given by Eq. (22), 
with $(\lambda, \alpha, \beta)=\left(\lambda_{1},-p,-p\right)$ for $m=1$, and $\left(\lambda_{3}, p, p\right)$ for $m=3$. The zero-energy Landau levels become

$$
\begin{gathered}
\epsilon_{n=-1}^{(1)}=\epsilon_{n=0}^{(1)}=-\frac{\gamma_{2}}{\sqrt{5}}, \\
\epsilon_{n=-1}^{(3)}=\epsilon_{n=0}^{(3)}=\frac{\gamma_{2}}{\sqrt{5}},
\end{gathered}
$$

which are valley degenerate, as they should be, due to spatial inversion symmetry. The two lowest levels of each bilayerlike block, $\epsilon_{n=-1}^{(m)}, \epsilon_{n=0}^{(m)}$, are split due to extra parameters as described by Eq. (23), so that the 16 zero-energy levels split into four energies, each of them with fourfold spin and valley degeneracy retained.

Figures 3(a) and 3(b) show the zero-field band structure and the Landau levels of four-layer graphene, respectively, computed numerically including all parameters. In Fig. 3(a), the dotted curve indicates the energy bands with the offdiagonal block neglected. The symbols $\mathrm{b}$ and $\mathrm{B}$ represent the light-mass bilayer $(m=1)$ and the heavy-mass bilayer block $(m=3)$, respectively. The Landau level spectrum is basically regarded as a composition of two series of bilayerlike levels, while there are anticrossings caused by $\mathcal{H}_{13}$ between the levels having the same index in modulo 3 . The valley degeneracy is never broken, even in the full parameter model, because it is protected by the spatial inversion symmetry of the lattice.

Figure 3(c) illustrates a two-dimensional map of the density of states calculated from the Landau levels in Fig. 3(b). The quantum Hall integer is always $4 M$ (where $M$ is an integer) because all the levels are valley and spin degenerate. Similarly to the trilayer, characteristic bright lines appear at crossing points of Landau levels belonging to the different bilayer blocks.

\section{FIVE-LAYER GRAPHENE}

The Hamiltonian of the ABA-stacked five-layer, Eq. (1) for $N=5$, may be partially decomposed into a monolayerlike block $m=0$ and two bilayerlike blocks with subsystem indices $m=2$ and $m=4$ :

$$
H_{N=5}=\left(\begin{array}{lcc}
\mathcal{H}_{0} & 0 & \mathcal{H}_{04} \\
0 & \mathcal{H}_{2} & 0 \\
\mathcal{H}_{40} & 0 & \mathcal{H}_{4}
\end{array}\right)
$$

where

$$
\begin{gathered}
\mathcal{H}_{0}=\left(\begin{array}{cc}
0 & v \pi^{\dagger} \\
v \pi & \Delta^{\prime}
\end{array}\right)-\frac{2}{3}\left(\begin{array}{cc}
\gamma_{2} & 0 \\
0 & \gamma_{5}
\end{array}\right), \\
\mathcal{H}_{2}=\mathcal{H}\left(\lambda_{2}\right)+\mathcal{W}(0,-1 / 2), \\
\mathcal{H}_{4}=\mathcal{H}\left(\lambda_{4}\right)+\mathcal{W}(2 / 3,1 / 2), \\
\mathcal{H}_{04}=\mathcal{H}_{40}^{\dagger}=[\mathcal{W}(\sqrt{2} / 6,0)]_{2 \times 4},
\end{gathered}
$$

where $\lambda_{2}=1, \lambda_{4}=\sqrt{3}$, and $[\mathcal{W}]_{2 \times 4}$ represents the upper half (i.e., the first two rows) of the matrix $\mathcal{W}$. The block $m=2$ is never mixed with $m=0$ and 4 due to the parity difference, as argued previously.
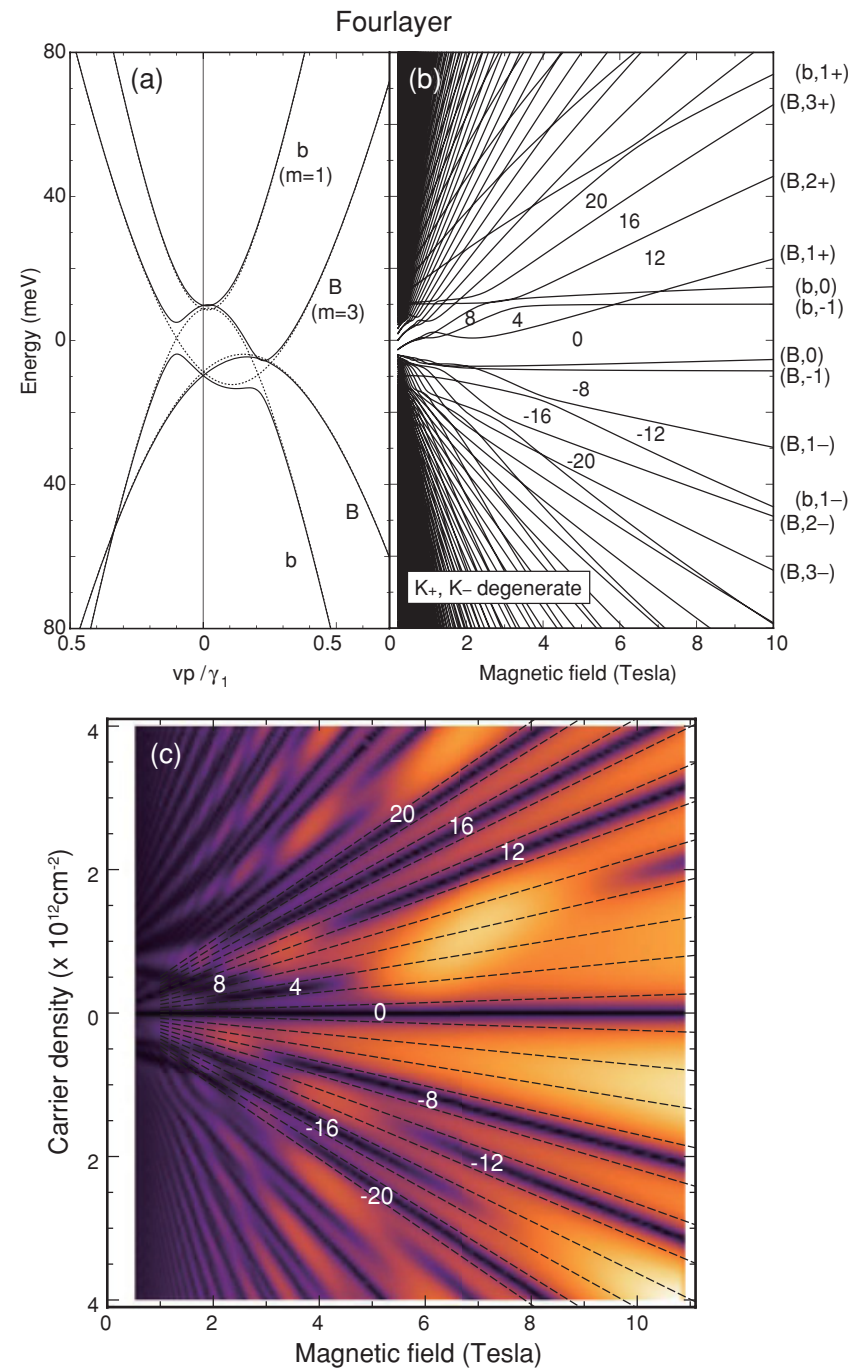

FIG. 3. (Color online) Plots similar to Fig. 2 for ABA four-layer graphene. In (a), the dotted curve indicates energy bands calculated by neglecting the off-diagonal block.

By neglecting the interblock mixing, $v_{3}$ and $v_{4}$, the zeroenergy Landau levels are given by

$$
\begin{gathered}
\epsilon_{n=0}^{(0)}=\frac{1+\xi}{2}\left(-\frac{\gamma_{2}}{2}\right)+\frac{1-\xi}{2}\left(\Delta^{\prime}-\frac{\gamma_{5}}{2}\right), \\
\epsilon_{n=-1}^{(2)}=\epsilon_{n=0}^{(2)}=-(1-\xi) \gamma_{2} \\
\epsilon_{n=-1}^{(4)}=\epsilon_{n=0}^{(4)}=\left(\frac{1+\xi}{3}+\frac{1-\xi}{4}\right) \gamma_{2} .
\end{gathered}
$$

Similarly to the trilayer and four-layer, the two lowest levels of each bilayerlike block, $\epsilon_{n=-1}^{(m)}, \epsilon_{n=0}^{(m)}$, split due to extra parameters in accordance with Eq. (23), resulting in 10 different zero-energy levels with spin degeneracy.

Figures 4(a) and 4(b) show the zero-field band structure and the Landau levels of five-layer graphene, respectively, numerically computed with all the parameters. Here the symbols $\mathrm{M}, \mathrm{b}$, and $\mathrm{B}$ represent the monolayer $(m=0)$, the light-mass bilayer $(m=2)$, and the heavy-mass bilayer block $(m=4)$, respectively. Figure 4(c) illustrates a two-dimensional map of the density of states and the quantum 

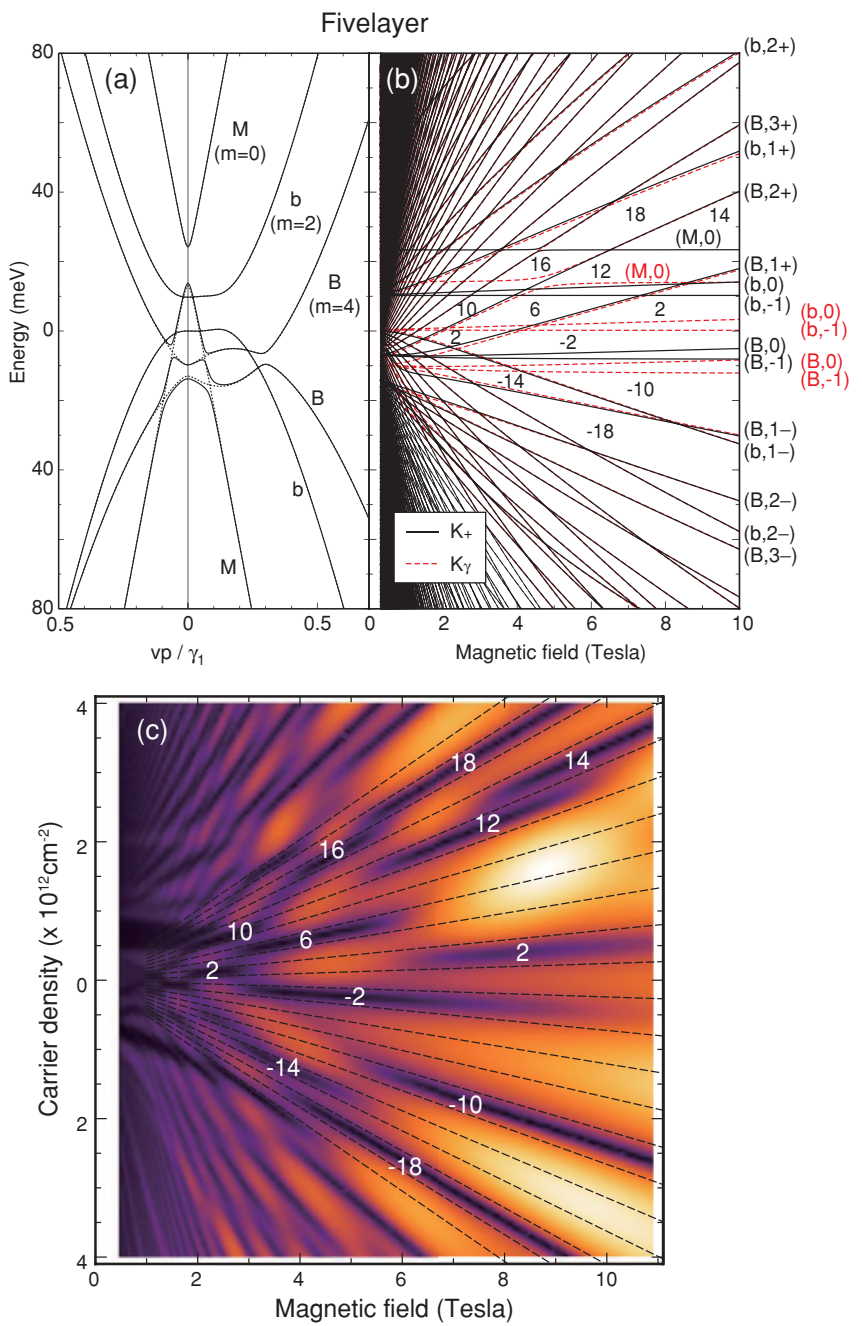

FIG. 4. (Color online) Plots similar to Fig. 2 for ABA five-layer graphene. In (a), the dotted curve indicates energy bands calculated by neglecting the off-diagonal block.
Hall conductivity. Similarly to the trilayer, the quantized Hall conductivity jumps by 2 only at the valley-split zero-energy levels of monolayerlike and bilayerlike subbands, whereas it changes in units of 4 at other levels which are almost valley degenerate. As a result, the quantized Hall conductivity becomes $4 M$ only in the region between monolayer zero levels of two valleys, and in the narrow gaps between -1 st and zeroth levels of each bilayer band. Otherwise it takes a series of $4 M+2$.

\section{CONCLUSION}

Here we used the effective-mass approximation to analyze the Landau level spectra of ABA-stacked multilayer graphenes. In general, the next-nearest layer couplings significantly influence the low-energy spectrum and the quantum Hall effect by causing energy shifts, level anticrossings, and valley splitting of the low-lying Landau levels, as described in detail here for trilayer, four-layer, and five-layer graphene. We considered a single-particle picture in order to provide a simple description of a broad range of features. Depending on sample quality, electron-electron interactions also contribute to symmetry breaking and Landau level splitting, ${ }^{67-79}$ as does interlayer asymmetry due to the presence of an external gate or doping. ${ }^{28,31,42,45,80-82}$ Nevertheless, an experimental observation of features related to next-nearest layer couplings should be possible in high-mobility samples including suspended graphene ${ }^{69,83-86}$ or graphene on a boron nitride substrate. ${ }^{66,72,73,87}$ In fact, Landau level crossings in trilayer graphene were recently observed, ${ }^{66}$ and they allowed the determination of Slonczewski-Weiss-McClure parameter values in remarkably close agreement with those of bulk graphite. ${ }^{32,88}$

\section{ACKNOWLEDGMENTS}

The authors thank T. Taychatanapat and P. Jarillo-Herrero for discussions and for sharing their experimental data prior to publication. This project has been funded by JST-EPSRC Japan-UK Cooperative Programme Grant No. EP/H025804/1.
${ }^{1}$ K. S. Novoselov, A. K. Geim, S. V. Morozov, D. Jiang, Y. Zhang, S. V. Dubonos, I. V. Grigorieva, and A. A. Firsov, Science 306, 666 (2004).

${ }^{2}$ K. S. Novoselov, A. K. Geim, S. V. Morozov, D. Jiang, M. I. Katsnelson, I. V. Grigorieva, S. V. Dubonos, and A. A. Firsov, Nature (London) 438, 197 (2005).

${ }^{3}$ Y. B. Zhang, Y. W. Tan, H. L. Stormer, and P. Kim, Nature (London) 438, 201 (2005).

${ }^{4}$ K. S. Novoselov, E. McCann, S. V. Morozov, V. I. Fal'ko, M. I. Katsnelson, U. Zeitler, D. Jiang, F. Schedin, and A. K. Geim, Nat. Phys. 2, 177 (2006).

${ }^{5}$ V. V. Cheianov and V. I. Fal'ko, Phys. Rev. B 74, 041403(R) (2006).

${ }^{6}$ M. I. Katsnelson, K. S. Novoselov, and A. K. Geim, Nat. Phys. 2, 620 (2006).

${ }^{7}$ B. Huard, J. A. Sulpizio, N. Stander, K. Todd, B. Yang, and D. Goldhaber-Gordon, Phys. Rev. Lett. 98, 236803 (2007).

${ }^{8}$ A. F. Young and P. Kim, Nat. Phys. 5, 222 (2009).
${ }^{9}$ A. F. Morpurgo and F. Guinea, Phys. Rev. Lett. 97, 196804 (2006).

${ }^{10}$ E. McCann, K. Kechedzhi, V. I. Fal'ko, H. Suzuura, T. Ando, and B. L. Altshuler, Phys. Rev. Lett. 97, 146805 (2006).

${ }^{11}$ S. V. Morozov, K. S. Novoselov, M. I. Katsnelson, F. Schedin, L. A. Ponomarenko, D. Jiang, and A. K. Geim, Phys. Rev. Lett. 97, 016801 (2006).

${ }^{12}$ H. B. Heersche, P. Jarillo-Herrero, J. Oostinga, L. M. K. Vandersypen, and A. F. Morpurgo, Nature (London) 446, 56 (2007).

${ }^{13}$ X. Wu, X. Li, Z. Song, C. Berger, and W. A. de Heer, Phys. Rev. Lett. 98, 136801 (2007).

${ }^{14}$ R. V. Gorbachev, F. V. Tikhonenko, A. S. Mayorov, D. W. Horsell, and A. K. Savchenko, Phys. Rev. Lett. 98, 176805 (2007).

${ }^{15}$ F. V. Tikhonenko, D. W. Horsell, R. V. Gorbachev, and A. K. Savchenko, Phys. Rev. Lett. 100, 056802 (2008).

${ }^{16}$ F. V. Tikhonenko, A. A. Kozikov, A. K. Savchenko, and R. V. Gorbachev, Phys. Rev. Lett. 103, 226801 (2009). 
${ }^{17}$ T. Ohta, A. Bostwick, T. Seyller, K. Horn, and E. Rotenberg, Science 313, 951 (2006)

${ }^{18}$ S. Y. Zhou, G.-H. Gweon, J. Graf, A. V. Fedorov, C. D. Spataru, R. D. Diehl, Y. Kopelevich, D.-H. Lee, S. G. Louie, and A. Lanzara, Nat. Phys. 2, 595 (2006).

${ }^{19}$ A. Bostwick, T. Ohta, T. Seyller, K. Horn, and E. Rotenberg, Nat. Phys. 3, 36 (2007).

${ }^{20}$ M. Mucha-Kruczyński, O. Tsyplyatyev, A. Grishin, E. McCann, V. I. Fal'ko, A. Bostwick, and E. Rotenberg, Phys. Rev. B 77, 195403 (2008).

${ }^{21}$ P. R. Wallace, Phys. Rev. 71, 622 (1947).

${ }^{22}$ J. C. Slonczewski and P. R. Weiss, Phys. Rev. 109, 272 (1958).

${ }^{23}$ J. W. McClure, Phys. Rev. 104, 666 (1956).

${ }^{24}$ N. H. Shon and T. Ando, J. Phys. Soc. Jpn. 67, 2421 (1998).

${ }^{25}$ Y. Zheng and T. Ando, Phys. Rev. B 65, 245420 (2002).

${ }^{26}$ V. P. Gusynin and S. G. Sharapov, Phys. Rev. Lett. 95, 146801 (2005).

${ }^{27}$ N. M. R. Peres, F. Guinea, and A. H. Castro Neto, Phys. Rev. B 73, 125411 (2006).

${ }^{28}$ E. McCann and V. I. Fal'ko, Phys. Rev. Lett. 96, 086805 (2006).

${ }^{29}$ F. Guinea, A. H. Castro Neto, and N. M. R. Peres, Phys. Rev. B 73, 245426 (2006).

${ }^{30}$ M. Koshino and T. Ando, Phys. Rev. B 73, 245403 (2006).

${ }^{31}$ E. V. Castro, K. S. Novoselov, S. V. Morozov, N. M. R. Peres, J. M. B. Lopes dos Santos, J. Nilsson, F. Guinea, A. K. Geim, and A. H. Castro Neto, Phys. Rev. Lett. 99, 216802 (2007).

${ }^{32}$ M. S. Dresselhaus and G. Dresselhaus, Adv. Phys. 51, 1 (2002).

${ }^{33}$ B. Partoens and F. M. Peeters, Phys. Rev. B 74, 075404 (2006); 75, 193402 (2007).

${ }^{34}$ C. L. Lu, C. P. Chang, Y. C. Huang, R. B. Chen, and M. L. Lin, Phys. Rev. B 73, 144427 (2006).

${ }^{35}$ S. Latil and L. Henrard, Phys. Rev. Lett. 97, 036803 (2006).

${ }^{36}$ M. Koshino and T. Ando, Phys. Rev. B 76, 085425 (2007); 77, 115313 (2008).

${ }^{37}$ H. Min and A. H. MacDonald, Phys. Rev. B 77, 155416 (2008).

${ }^{38}$ M. Koshino and T. Ando, Solid State Commun. 149, 1123 (2009).

${ }^{39}$ M. Aoki and H. Amawashi, Solid State Commun. 142, 123 (2007).

${ }^{40}$ M. Nakamura and L. Hirasawa, Phys. Rev. B 77, 045429 (2008).

${ }^{41}$ M. Nakamura, L. Hirasawa, and K. Imura, Phys. Rev. B 78, 033403 (2008).

${ }^{42}$ M. Koshino and E. McCann, Phys. Rev. B 79, 125443 (2009).

${ }^{43}$ A. A. Avetisyan, B. Partoens, and F. M. Peeters, Phys. Rev. B 79, 035421 (2009).

${ }^{44}$ A. A. Avetisyan, B. Partoens, and F. M. Peeters, Phys. Rev. B 80, 195401 (2009).

${ }^{45}$ M. Koshino and E. McCann, Phys. Rev. B 81, 115315 (2010).

${ }^{46}$ T. Ohta, A. Bostwick, J. L. McChesney, T. Seyller, K. Horn, and E. Rotenberg, Phys. Rev. Lett. 98, 206802 (2007).

${ }^{47}$ J. Güttinger, C. Stampfer, F. Molitor, D. Graf, T. Ihn, and K. Ensslin, New J. Phys. 10, 125029 (2008).

${ }^{48}$ M. F. Craciun, S. Russo, M. Yamamoto, J. B. Oostinga, A. F. Morpurgo, and S. Tarucha, Nat. Nanotechnol. 4, 383 (2009).

${ }^{49}$ W. Zhu, V. Perebeinos, M. Freitag, and P. Avouris, Phys. Rev. B 80, 235402 (2009).

${ }^{50}$ C. H. Lui, Z. Li, Z. Chen, P. V. Klimov, L. E. Brus, and T. F. Heinz, Nano Lett. 11, 164 (2011).

${ }^{51}$ Z. Jiang, E. A. Henriksen, L. C. Tung, Y.-J. Wang, M. E. Schwartz, M. Y. Han, P. Kim, and H. L. Stormer, Phys. Rev. Lett. 98, 197403 (2007).
${ }^{52}$ J. Martin, N. Akerman, G. Ulbricht, T. Lohmann, J. H. Smet, K. von Klitzing, and A. Yacoby, Nat. Phys. 4, 144 (2007).

${ }^{53}$ J. Yan, E. A. Henriksen, P. Kim, and A. Pinczuk, Phys. Rev. Lett. 101, 136804 (2008).

${ }^{54}$ L. M. Zhang, Z. Q. Li, D. N. Basov, M. M. Fogler, Z. Hao, and M. C. Martin, Phys. Rev. B 78, 235408 (2008).

${ }^{55}$ Z. Q. Li, E. A. Henriksen, Z. Jiang, Z. Hao, M. C. Martin, P. Kim, H. L. Stormer, and D. N. Basov, Phys. Rev. Lett. 102, 037403 (2009).

${ }^{56}$ K. F. Mak, C. H. Lui, J. Shan, and T. F. Heinz, Phys. Rev. Lett. 102, 256405 (2009).

${ }^{57}$ A. B. Kuzmenko, I. Crassee, D. van der Marel, P. Blake, and K. S. Novoselov, Phys. Rev. B 80, 165406 (2009).

${ }^{58}$ E. A. Henriksen and J. P. Eisenstein, Phys. Rev. B 82, 041412 (2010).

${ }^{59}$ Corners of the hexagonal Brillouin zone are located at wave vector $\mathbf{K}_{\xi}=\xi\left(\frac{4}{3} \pi a^{-1}, 0\right)$, where $\xi= \pm 1$ and $a$ is the lattice constant.

${ }^{60}$ J. L. Manes, F. Guinea, and M. A. H. Vozmediano, Phys. Rev. B 75, 155424 (2007)

${ }^{61}$ J. R. Schrieffer and P. A. Wolff, Phys. Rev. 149, 491 (1966).

${ }^{62}$ M. Koshino and E. McCann, Phys. Rev. B 80, 165409 (2009).

${ }^{63}$ The Quantum Hall Effect, edited by R. E. Prange and S. M. Girvin (Springer-Verlag, New York, 1986).

${ }^{64}$ M. Koshino and T. Ando, Phys. Rev. B 81, 195431 (2010).

${ }^{65}$ T. Ando and Y. Uemura, J. Phys. Soc. Jpn. 36, 959 (1974).

${ }^{66} \mathrm{~T}$. Taychatanapat, K. Watanabe, T. Taniguchi, and P. Jarillo-Herrero (unpublished).

${ }^{67}$ Y. Zhang, Z. Jiang, J. P. Small, M. S. Purewal, Y.-W. Tan, M. Fazlollahi, J. D. Chudow, J. A. Jaszczak, H. L. Stormer, and P. Kim, Phys. Rev. Lett. 96, 136806 (2006).

${ }^{68}$ A. J. M. Giesbers, L. A. Ponomarenko, K. S. Novoselov, A. K. Geim, M. I. Katsnelson, J. C. Maan, and U. Zeitler, Phys. Rev. B 80, 201403 (2009).

${ }^{69}$ B. E. Feldman, J. Martin, and A. Yacoby, Nat. Phys. 5, 889 (2009).

${ }^{70}$ Y. Zhao, P. Cadden Zimansky, Z. Jiang, and P. Kim, Phys. Rev. Lett. 104, 066801 (2010).

${ }^{71}$ X. Du, I. Skachko, F. Duerr, A. Luican, and E. Y. Andrei, Nature (London) 462, 192 (2009).

${ }^{72}$ C. R. Dean, A. F. Young, I. Meric, C. Lee, L. Wang, S. Sorgenfrei, K. Watanabe, T. Taniguchi, P. Kim, K. L. Shepard, and J. Hone, Nat. Nanotechnol. 5, 722 (2010).

${ }^{73}$ C. R. Dean, A. F. Young, P. Cadden Zimansky, L. Wang, H. Ren, K. Watanabe, T. Taniguchi, P. Kim, J. Hone, and K. L. Shepard, e-print arXiv:1010.1179 (to be published).

${ }^{74}$ H. Min, G. Borghi, M. Polini, and A. H. MacDonald, Phys. Rev. B 77, 041407 (R) (2008).

${ }^{75}$ R. Nandkishore and L. Levitov, Phys. Rev. Lett. 104, 156803 (2010).

${ }^{76}$ F. Zhang, H. Min, M. Polini, and A. H. MacDonald, Phys. Rev. B 81, 041402 (R) (2010).

${ }^{77}$ O. Vafek and K. Yang, Phys. Rev. B 81, 041401(R) (2010).

${ }^{78}$ R. Nandkishore and L. Levitov, Phys. Rev. B 82, 115124 (2010).

${ }^{79}$ Y. Lemonik, I. L. Aleiner, C. Toke, and V. I. Falko, Phys. Rev. B 82, 201408 (2010).

${ }^{80}$ E. McCann, Phys. Rev. B 74, 161403(R) (2006). 
${ }^{81}$ M. Mucha-Kruczyński, E. McCann, and V. I. Fal'ko, Solid State Commun. 149, 1111 (2009).

${ }^{82}$ L. M. Zhang, M. M. Fogler, and D. P. Arovas, e-print arXiv:1008.1418 (to be published).

${ }^{83}$ K. I. Bolotin, K. J. Sikes, Z. Jiang, M. Klima, G. Fudenberg, J. Hone, P. Kim, and H. L. Stormer, Solid State Commun. 146, 351 (2008).

${ }^{84}$ X. Du, I. Skachko, A. Barker, and E. Y. Andrei, Nat. Nanotechnol. 3, 491 (2008).

${ }^{85}$ K. I. Bolotin, K. J. Sikes, J. Hone, H. L. Stormer, and P. Kim, Phys. Rev. Lett. 101, 096802 (2008).
${ }^{86}$ W. Bao, Z. Zhao, H. Zhang, G. Liu, P. Kratz, L. Jing, J. Velasco Jr., D. Smirnov, and C. N. Lau, Phys. Rev. Lett. 105, 246601 (2010).

${ }^{87}$ A. F. Young, C. R. Dean, I. Meric, S. Sorgenfrei, H. Ren, K. Watanabe, T. Taniguchi, J. Hone, K. L. Shepard, and P. Kim, e-print arXiv:1004.5556 (to be published).

${ }^{88}$ Recent experiments (Ref. 66) on high-mobility trilayer samples determined Slonczewski-Weiss-McClure parameter values to be $\gamma_{2}=-0.028 \mathrm{eV}, \gamma_{4}=0.041 \mathrm{eV}, \gamma_{5}=0.05 \mathrm{eV}$ and $\Delta^{\prime}=0.046$ $\mathrm{eV}$, when $\gamma_{0}=3.1 \mathrm{eV}, \gamma_{1}=0.39 \mathrm{eV}$ and $\gamma_{3}=0.315 \mathrm{eV}$ are assumed. 